\title{
Compensating Power Depletion due to Stimulated Raman Scattering in High-Power Delivery Fiber via Spectral Inversion Revisited in A View of Experimental Implementation
}

\author{
Peng Dong \\ Department of fundamental physics, China University of Petroleum (East China), Qingdao 266580, China \\ Corresponding to: dongpeng@upc.edu.cn
}

\begin{abstract}
Broadband spectral inversion was proved theoretically to be an effective method to compensate power depletion due to stimulated Raman scattering in high-power delivery fiber. A critical difficulty in implementing the method in experiment is to realize broadband spectral inversion of incoherent light as Raman Stokes waves are incoherent due to their origin from spontaneous emission noise. Broadband spectral inversion of incoherent light is investigated experimentally in this article. A beam from an amplified spontaneous emission (ASE) light source is used as an approximated Raman Stokes waves in the experiment. ASE Spectrum of width of $10.5 \mathrm{~nm}$ is inverted via four-wave mixing in a highly nonlinear dispersion-shifted fiber in efficiency of -10dB without significant spectrum deformation. A theoretical model for four-wave mixing of ASE incoherent light is established, and based on which the limitation in more broadband spectral inversion of ASE incoherent light is analyzed.
\end{abstract}

\section{Keywords}

Polarization Spectral inversion; four-wave mixing; incoherent light; amplified spontaneous emission

\section{Academic Discipline And Sub-Disciplines}

Physics; Optics; Nonlinear fiber optics

\section{SUBJECT CLASSIFICATION}

Physics

\section{INTRODUCTION}

Raman scattering is a third-order nonlinear effect in which the signal is scattered into the Stokes waves at downshifted frequencies. In fiber, a spontaneous Raman scattering becomes stimulated at a threshold value (which is about $600 \mathrm{~mW}$ for a typical single-mode fiber at a communication wavelength of $1.55 \mu \mathrm{m}[1]$ ) and the signal rapidly transfers its power to the Stokes waves. High power fiber lasers and amplifiers with dramatically growing output power up to $20 \mathrm{~kW}$ for continuous wave[2] and MG watts order for pulsed wave[3,4] have been developed rapidly in recent years to meet application requirements from industry, medicine, military and so on. The occurrence of stimulated Raman scattering (SRS) has been a crucial limiting factor for fibers to deliver such a high power from the high power fiber lasers or amplifiers to the application fields mentioned above.

In the past ten years, several research groups suggested different methods to avoid or suppress SRS in highpower fiber systems. One of the main methods is to adopt micro-structured fibers with designed filter functions [5-8], which however makes the fiber manufacturing process more complicated. Our group proposed a method for compensating power depletion due to SRS in high-power delivery fiber via broadband spectral inversion[9]. This method was proved theoretically to be promising. A critical difficulty in implementing the method in experiment is to realize broadband spectral inversion. As Raman Stokes waves are from spontaneous emission noise, the broadband spectral inversion should be performed on incoherent light.

Spectral inversion is an essential technique in WDM networks[10]. There are many methods to implement alloptical spectral inversion, such as cross-gain modulation[11], cross-phase modulation[12-14] and four-wave mixing (FWM)[15,16], among which FWM in fibers is regarded as a promising one due to its numerous 
advantages, e.g. high speed, low noise, easy to be integrated, broad bandwidth[17].

Broadband spectral inversion of incoherent light via FWM is seldom reported. In recent years, our group demonstrated spectral inversion of a tunable incoherent signal with $2 \mathrm{~nm}$ bandwidth in 2006[18] and simultaneous spectral inversion of 22 incoherent signal channels with totality of bandwidth of $17 \mathrm{~nm}$ in 2009[19] respectively. In these works, a theoretical model of FWM between coherent pump and incoherent signal was adopted and based on which the experimental results were well explained, however, the time feature of occurrence of FWM in fibers and characteristic time of random phase fluctuation of incoherent light are not given a close examination.

In this paper, Broadband spectral inversion of incoherent light is investigated experimentally to provide an implementation solution for compensating power depletion due to SRS in high-power delivery fiber. Firstly, experiment on broadband spectral inversion of incoherent light via FWM is demonstrated using a beam from an amplified spontaneous emission (ASE) light source as broadband incoherent signal. Secondly, a theoretical model of FWM between coherent pump and ASE incoherent light is established after making a close examination on the time feature of occurrence of FWM in fibers and characteristic time of random phase fluctuation of ASE light. At last, the prospect and limitation in realizing broadband spectral inversion of ASE incoherent light is analyzed.

\section{EXPERIMENT DEMONSTRATION}

\subsection{Experiment demonstration}

As it is explained in the introduction section, the broadband spectral inversion of incoherent light to be realized in this article aims to provide an implementation solution for compensating power depletion due to SRS in high-power delivery fiber reported in Ref. 9. The light beam needing to be spectrally inverted in Ref. 9 includes a signal transmitted in fiber, which may be coherent or incoherent according to the specific application background, and Raman stokes light of the signal, which is incoherent light as it is seeded by spontaneous emission noise. As a whole, we take the light beam needing to be spectrally inverted as incoherent light, and use an ASE light source as an approximated replacement of it.

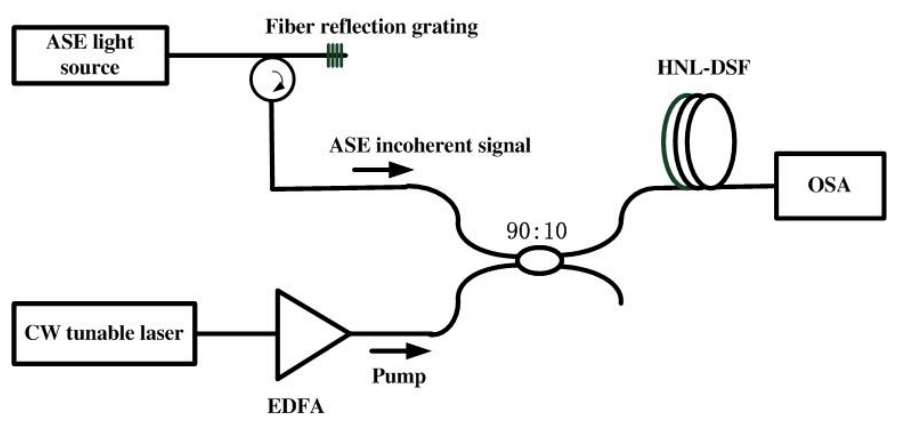

Fig.1 Experimental setup for spectral inversion of broadband ASE incoherent signal light. (ASE: Amplified Spontaneous Emission; CW: Continuous Wave; EDFA: $\mathrm{Er}^{3+}$-doped Fiber Amplifier; HNL-DSF: Highly Nonlinear dispersion-shifted Fiber; OSA: Optical Spectrum Analyzer.)

The experimental setup is shown in Fig.1. The incoherent signal is provided by an ASE broadband light source (Opticwave BLS-CL-13) filtered by a fiber reflection grating. The ASE light has a $-10 \mathrm{dBm}$-bandwidth of $1527.8 \sim 1564.5 \mathrm{~nm}$. The fiber reflection grating has a reflection bandwidth of $10.5 \mathrm{~nm}$ from $1544.5 \mathrm{~nm}$ to $1555.0 \mathrm{~nm}$. The spectrum of the ASE light source and that of the filtered ASE signal from the fiber reflection grating are shown in Fig. 2. 


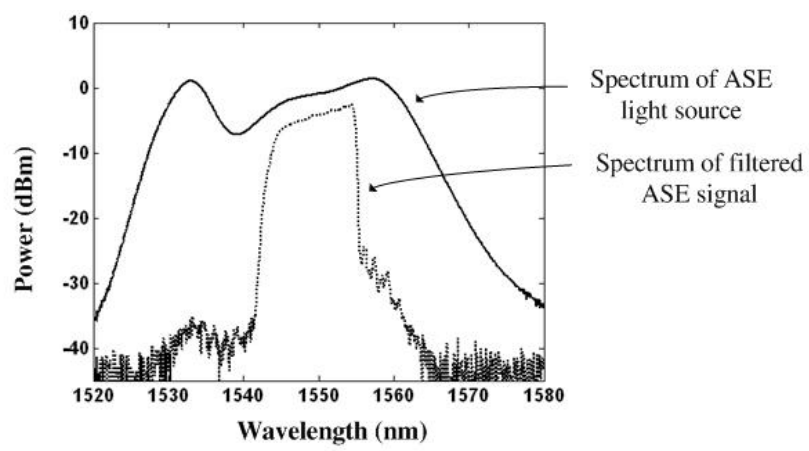

Fig.2 Spectra of ASE light source and the filtered ASE signal light.

A continuous wave (CW) laser (Agilent 8164A) provides the pump light at a wavelength of $1543.4 \mathrm{~nm}$, which is near to the zero dispersion wavelength (ZDW) of the highly nonlinear dispersion-shifted fiber (HNL-DSF) (1543nm). The pump light after being amplified by an $\mathrm{Er}^{3+}$-doped fiber amplifier (EDFA) together with the ASE incoherent signal are multiplied by a 90:10 coupler and launched into the HNL-DSF for degenerate FWM between coherent pump light and broadband ASE incoherent light. The HNL-DSF is $1 \mathrm{~km}$ long and has a high nonlinear coefficient of $10 \mathrm{~W}^{-1} \mathrm{~km}^{-1}$.

The spectral inversion realized via FWM in HNL-DSF is measured by an optical spectrum analyzer (OSA).

\subsection{Experimental results}

The experimental result of spectral inversion of the ASE incoherent signal with bandwidth of $10.5 \mathrm{~nm}$ is given in Fig.3. The pump power after the coupler is measured to be $30 \mathrm{dBm}$. The central-wavelength power of the ASE incoherent signal is measured to be $-4.2 \mathrm{dBm}$. As shown in Fig.3, the ASE incoherent signal is spectrally inverted and its spectrum is well copied by the idle signal.

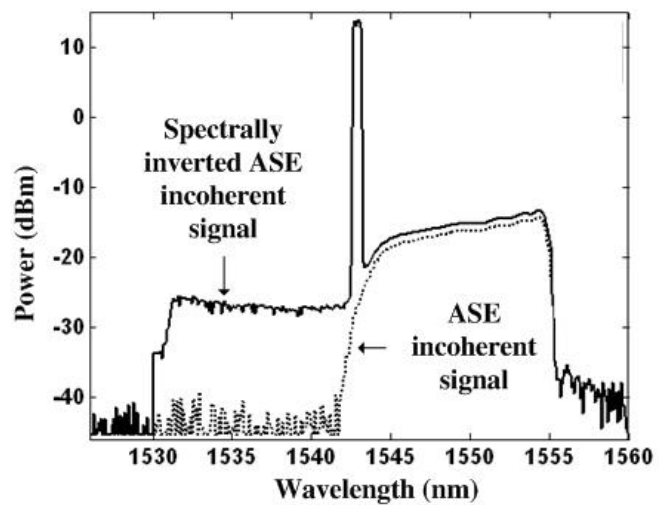

Fig.3 Experimental result of spectral inversion of ASE incoherent signal light with bandwidth of $10.5 \mathrm{~nm}$.

We define the ratio of the output idle light power with respect to the input signal power as the conversion efficiency, which is described as

$$
\eta=10 \log \left(\left|A_{i}(L)\right|^{2} /\left|A_{s}(0)\right|^{2}\right)
$$

where $A_{s}(0)$ and $A_{i}(\mathrm{~L})$ are complex amplitudes of input signal and output idle light at a fiber length of $L$. The spectral inversion efficiency shown in Fig. 3 is about $-10 \mathrm{~dB}$.

\section{Theoretical Model of FWM Between a Coherent Pump and ASE Incoherent Light}

The electrical field of the coherent laser pump is expressed as 


$$
E_{p}(z, t)=A_{p} \exp \left[j\left(\varpi_{p} t-\beta_{p} z\right)\right]
$$

Compared with coherent light, incoherent light has broader bandwidth and randomly fluctuated amplitude and phase. For simplicity, assume the amplitude does not vary with time, thus the electrical field of incoherent light is represented by

$$
E_{s}(z, t)=\int A_{s}\left(z, \varpi_{s}\right) \exp \left[j\left(\varpi_{s} t-\beta_{s} z\right)+i \varphi\left(\varpi_{s}, t\right)\right] d \varpi_{s}
$$

where, $A_{s}\left(z, \omega_{s}\right)$ represents the amplitude of frequency component $\omega_{s}$ which does not change with time as assumption. $\varphi\left(\omega_{s,} t\right)$ represents the phase of frequency component $\omega_{s,}$ which varies with frequency and fluctuates with time. For partially coherent light, $\varphi\left(\omega_{s,} t\right)$ follows Gaussian statistic distribution in time according to phase-diffusion model ${ }^{[20]}$.

Firstly, the impact of phase fluctuation of incoherent light on the occurrence of FWM is analyzed. For any frequency component $\omega_{s,}$ we make a close examination on the characteristic time of phase fluctuation. If the incoherent light specifically is ASE light, the characteristic time of phase fluctuation is in the order of fluorescence lifetime of $10^{-9} \mathrm{~s}$, which is much longer than the occurrence time of FWM in fiber. The mechanism of FWM in fiber is the distortion of electron cloud in the material of fiber, which has fast response time of about $10^{-15} \sim 10^{-16} \mathrm{~s}$. Therefore, for any frequency component $\omega_{s}$ of the ASE incoherent light, its phase can be thought to be invariable at the "instant" of the occurrence of FWM in fiber, showing a feature of coherent light in the process of FWM. Thus, the FWM of this frequency can be calculated according to the coupled-wave equations describing the FWM of coherent light. Considering the relatively slow fluctuation of the phase of ASE incoherent light, the calculation results of each "instant" need to be statistically averaged during a period longer than the phase.

Secondly, the impact of broad bandwidth of the incoherent signal light on FWM is analyzed. FWM happens between a broadband signal light and the pump light. It is worth noting that the pump light pumps all the frequency components simultaneously. A simplified method for calculating FWM is that calculating FWM between each frequency component and pump light respectively at first and then integrating the calculated results of all the frequency components of the incoherent signal light without taking the depletion of pump into consideration. Ref.18 adopted this method since the bandwidth of the incoherent light is only $2 \mathrm{~nm}$. However, in the case of broad bandwidth, the depletion of pump is prominent and cannot be neglected, thus the FWM between each frequency component and pump should be calculated simultaneously. In such a case, the coupled-wave equations of FWM are expressed as follows:

$$
\begin{gathered}
\frac{d A_{p}}{d z}=i \gamma\left[\left|A_{p}\right|^{2}+\sum_{m=1}^{M} 2\left(\left|A_{s}^{m}\right|^{2}+\left|A_{i}^{m}\right|^{2}\right)\right] A_{p}+2 i \gamma \sum_{m=1}^{M} A_{p}^{*} A_{s}^{m} A_{i}^{m} e^{i \Delta \beta^{m} z}-\frac{1}{2} \alpha_{p} A_{p}^{\prime} \\
\frac{d A_{s}^{m}}{d z}=i \gamma\left[\left|A_{s}^{m}\right|^{2}+2\left(\left|A_{i}^{m}\right|^{2}+\left|A_{p}\right|^{2}\right)\right] A_{s}^{m}+i \gamma A_{p}^{2}\left(A_{i}^{m}\right)^{*} e^{-i \Delta \beta^{m} z}-\frac{1}{2} \alpha_{s}^{m} A_{s}^{m}, \\
\frac{d A_{i}^{m}}{d z}=i \gamma\left[\left|A_{i}^{m}\right|^{2}+2\left(\left|A_{s}^{m}\right|^{2}+\left|A_{p}\right|^{2}\right)\right] A_{i}^{m}+i \gamma A_{p}^{2}\left(A_{s}^{m}\right)^{*} e^{-i \Delta \beta^{m} z}-\frac{1}{2} \alpha_{i}^{m} A_{i}^{m},
\end{gathered}
$$

where $A_{p \prime} A_{s,} A_{i}$ are the complex amplitudes of pump, signal and idle light, respectively, $m$ represents the frequency component of incoherent signals, and $\Delta \beta$ is the phase mismatch given by

$$
\begin{aligned}
& \Delta \beta=\beta_{s}^{m}+\beta_{i}^{m}-2 \beta_{p} \\
& =\frac{\lambda_{0}{ }^{4}}{24 \pi^{2} c^{2}} \frac{d D_{c}}{d \lambda}\left[\left(\varpi_{s}^{m}-\varpi_{0}\right)^{3}+\left(\varpi_{i}^{m}-\varpi_{0}\right)^{3}-2\left(\varpi_{p}-\varpi_{0}\right)^{3}\right] \\
& -\left(\frac{\lambda_{0}{ }^{5}}{32 \pi^{3} c^{3}} \frac{d D_{c}}{d \lambda}+\frac{\pi \lambda_{0}{ }^{6}}{192 c^{3}} \frac{d^{2} D_{c}}{d \lambda^{2}}\right) \times\left[\left(\varpi_{s}^{m}-\varpi_{0}\right)^{4}+\left(\varpi_{i}^{m}-\varpi_{0}\right)^{4}-2\left(\varpi_{p}-\varpi_{0}\right)^{4}\right]
\end{aligned}
$$


where $\omega_{0}$ is the frequency at which the second-order dispersion coefficient of the HNL-DSF is zero, $\omega_{p,} \omega_{s,}$ and $\omega_{i}$ are circular frequencies of pump, signal and idle light respectively.

The sum in equation (4.1) represents the simultaneous action of different frequency components of the incoherent signal light.

The above analysis shows the principle of the FWM between a coherent pump and incoherent signals. As a special example, when only one frequency component of the incoherent signals is considered, the incoherent FWM will degenerate to the coherent FWM.

In our work reported in Ref. 19, although without making a close examination on the feature of FWM between coherent pump light and ASE incoherent signal channels, we adopted the above coupled wave equations (4.1) $\sim(4.4)$ and the theoretical results agree well with the experimental results.

According to the theoretical model built above, it can be inferred that the bandwidth of ASE incoherent light which can be spectrally inverted in experiment is mainly limited by the bandwidth of the ASE incoherent signal light.

\section{Conclusion}

In this paper, Broadband spectral inversion of incoherent light is investigated experimentally. Spectral inversion has been successfully achieved in experiment on an ASE incoherent light of bandwidth of $10.5 \mathrm{~nm}$ with efficiency of $-10 \mathrm{~dB}$. A theoretical model of FWM between a coherent pump light and a broadband ASE incoherent signal light is built after making a close examination on the time feature of occurrence of FWM in fibers and characteristic time of random phase fluctuation of ASE light, based on which it can be inferred that the bandwidth of ASE incoherent light which can be spectrally inverted in experiment is mainly limited by the bandwidth of the ASE light source. The realization of broadband spectral inversion of incoherent light provides an implementation solution for compensating power depletion due to SRS in high-power delivery fiber.

\section{REFERENCES}

1. G. P. Agrawal, 2013,Nonlinear Fiber Optics, Fifth Edition,Academic Press.

2. V. Fomin, A. Ferin, M. Abramov, I. Samartsev, and V. Gapontsev, 2014 Ultra-high power single mode fiber laser system with non-uniformly configure fiber-to-fiber rod multimode amplifier, US Patent 20140314106 A1.

http://xueshu.baidu.com/s?wd=paperuri\%3A\%281ed8d866ec285f38235786d624f8dc1a\%29\&filter=sc lon g sign\&tn=SE xueshusource $2 \mathrm{kduw} 22 \mathrm{v} \& \mathrm{sc}$ vurl=http\%3A\%2F\%2Fwww.freepatentsonline.com\%2Fy2014 $\% 2 F 0314106 . h t m \mid \& i e=u t f-8 \&$ sc us $=14114047507174538137$

3. A. Klenke, S. Hädrich, T. Eidam, J. Rothhardt, M. Kienel, S. Demmler, T. Gottschall, J. Limpert, and A. Tünnermann, 201422 GW peak-power fiber chirped-pulse-amplification system, Opt. Lett. 39(24), 68756878.

\section{https://doi.org/10.1364/OL.39.006875}

4. P. S. Teh, R. J. Lewis, S. U. Alam, and D. J. Richardson, 2013200 W Diffraction limited, single-polarization, all-fiber picosecond MOPA, Opt. Express 21(22), 25883-25889.

\section{https://doi.org/10.1364/OE.21.025883}

5. L. A. Zenteno, J. Wang, D. T. Walton, B. A. Ruffin, M. J. Li, S. Gray, A. Crowley and X. Chen, 2005 Suppression of Raman gain in single-transverse-mode dual-hole assisted fiber, Opt. Express 13(22), 89218926. 
https://doi.org/10.1364/OPEX.13.008921

6. J. M. Fini, M. D. Mermelstein, M. F. Yan, R. T. Bise, A. D. Yablon, P. W. Wisk, and M. J. Andrejco, 2006 Distributed suppression of stimulated Raman scattering in an Yb-doped filter-fiber amplifier, Opt. Lett. 31(17), 2550-2552.

\section{https://doi.org/10.1364/OL.31.002550}

7. J. Kim, P. Dupriez, C. Codemard, J. Nilsson, and J. K. Sahu, 2006 Suppression of stimulated Raman scattering in a high power $\mathrm{Yb}$-doped fiber amplifier using a W-type core with fundamental mode cut-off, Opt. Express 14 (12), 5103-5113.

\section{https://doi.org/10.1364/OE.14.005103}

8. A. Shirakawa, Y. Suzuki, S. Arisa, M. Chen, C. B. Olausson, J. K. Lyngso, and J. Broeng, 2013 High-peak power pulse amplification by SRS-suppressed photonic bandgap fiber, in Proceedings of the Pacific Rim Conf., Lasers Electro-Optics, CLEO - Tech. Digest 7-8.

http://xueshu.baidu.com/s?wd=paperuri\%3A\%28b354e347b29a06bea2fa3f6509081382\%29\&filter=sc lon g sign\&tn=SE xueshusource $2 \mathrm{kduw} 22 \mathrm{v} \& \mathrm{sc}$ vurl=http\%3A\%2F\%2Fwww.opticsinfobase.org\%2Fabstract.cf $\underline{\text { m\%3Furi\%3DCLEOPR-2013-TuA2 1\&ie }=u t f-8 \& s c \text { us }=13421046352820590469}$

9. P. Dong, X. Xiao, Y. Tian, S. Gao, and C. Yang, 2008 Compensating power depletion due to stimulated Raman scattering in high-power delivery fiber via spectral inversion, J. Opt. Soc. Am. B, 25 (1), 48-53.

https://doi.org/10.1364/JOSAB.25.000048

10. S. J. B. Yoo, Wavelength conversion technologies for WDM network applications, 1996 J. Lightwave Technol., 14 (6), 955-966

http://ieeexplore.ieee.org/stamp/stamp.jsp?arnumber $=511595$

11. O. Qasaimeh, 2004 Characteristics of cross-gain (XG) wavelength conversion in quantum dot semiconductor optical amplifiers, IEEE Photon. Technol. Lett. , 16 (2), 542-544

http://ieeexplore.ieee.org/document/1266484/

12. A. S. Lenihan, R. Salem, T. E. Murphy, and G. M. Carter, 2006 All-optical 80-gb/s time-division demultiplexing using polarization-insensitive cross-phase modulation in photonic crystal fiber, IEEE Photon. Technol. Lett., 18(12), 1329-1331

http://ieeexplore.ieee.org/document/1637712/?arnumber=1637712

13. H. Tsuchida, T. Simoyama, H. Ishikawa, T. Mozume, M. Nagase, and J.-i. Kasai, 2007 Cross-phasemodulation-based wavelength conversion using intersubband transition in InGaAs/AIAs/AIAsSb coupled quantum wells. Opt. Lett. 32(7), 751-753

https://doi.org/10.1364/OL.32.000751

14. D. Krcmarik, M. Karasek, J. Radil, and J. Vojtech, 2007 Multi-wavelength conversion at $10 \mathrm{~Gb} / \mathrm{s}$ using crossphase modulation in highly nonlinear fiber, Optics Commu., 278(2) , 402-412

https://doi.org/10.1016/j.optcom.2007.06.004

15. P. A. Andersen, T. Tokle, G. Yan, C. Peucheret, and P. Jeppesen, 2005 Wavelength conversion of a 40-Gb/s RZ-DPSK signal using four-wave mixing in a dispersion-flattened highly nonlinear photonic crystal fiber, IEEE Photon. Technol. Lett., 17, 1908-1910 
http://xueshu.baidu.com/s?wd=paperuri\%3A\%28b8fdafe93cc08c838987934af22593dc\%29\&filter=sc long sign\&tn=SE xueshusource 2kduw22v\&sc vurl=http\%3A\%2F\%2Fieeexplore.ieee.org\%2Fdocument\%2F14 98899\%2F\&ie $=$ utf-8\&sc us $=18216222931410859594$

16. P. Devgan, R. Tang, V. S. Grigoryan, and P. Kumar, 2006 Highly efficient multichannel wavelength conversion of DPSK signals, J. Lightwave Technol., 24(10), 3677-3682

https://www.osapublishing.org/jlt/abstract.cfm?uri=jlt-24-10-3677

17. J. Hansryd, P. A. Andrekson, M. Westlund, L. Jie, and P. O. Hedekvist, 2002 Applications of fiber-based optical parametric amplifiers, IEEE Journal of Selected Topics in Quantum Electronics, 8, 506-520

http://ieeexplore.ieee.org/document/1027573/?arnumber=1027573

18. S. Gao, C. Yang, X. Xiao, Y. Tian, Z. You, and G. Jin, 2006 Wavelength conversion of spectrum-sliced broadband amplified spontaneous emission light by hybrid four-wave mixing in highly nonlinear, dispersion-shifted fibers, Opt. Express, 14(7) , 2873-2879

\section{https://doi.org/10.1364/OE.14.002873}

19. P. Dong, Y. Tian, X. Xiao, and C. Yang, 2012 Wavelength conversion of multichannel spectrum-sliced ASE signals via four-wave mixing in highly nonlinear dispersion-shifted fiber, J. Nonlinear Opt. Phys. Mater. 18 (2) $341-348$

https://doi.org/10.1142/S0218863509004658

\section{Author' biography with Photo}

Peng Dong is a lecturer of China University of Petroleum(East China), Qingdao, China. Her research interests include nonlinear optical effects in fibers, supercontinuum generation in fibers, nonlinear optical effects in high power fiber lasers and so on.

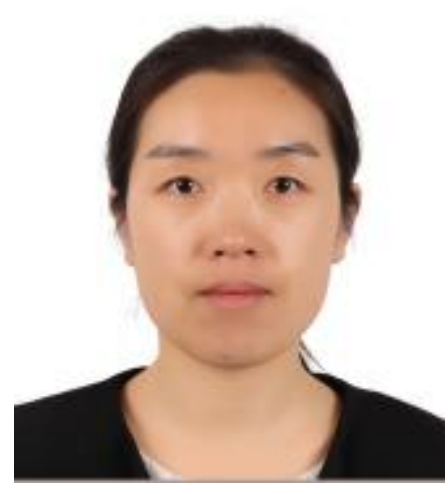

